\title{
A Multi-Axis Force Measurement System for a Space Docking Mechanism
}

\author{
Gangfeng Liu ${ }^{\mathrm{a}}$, Changle $\mathrm{Li}^{\mathrm{b}}$ and Zenghui $\mathrm{Xie}^{\mathrm{c}}$ \\ Building C1, State Key Laboratory of Robotics and Systems, Harbin Institute of Technology, Harbin, \\ 150001, China

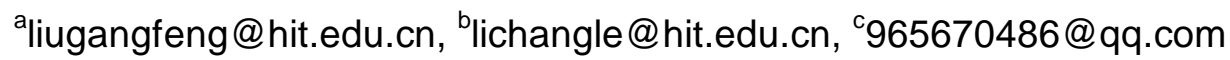

Keywords: multi-axis force measurement system, 6-UPS parallel mechanism, impedance control system.

\begin{abstract}
In order to ensure the security buffer of the 6-UPS space docking mechanism, real-time control based on contact force is needed. In this paper, a highly effective multi-axis force measurement system is built to acquire and analyze the real time force from six separate force sensors. According to the data analysis of the measurement system, a compound contact force is used as the feedback signal to control the movement of the space docking mechanism. Ground experiment shows the good effect of the measurement system, and that the force control algorithm improves the buffer effect at the docking stage.
\end{abstract}

\section{Introduction}

The 6-UPS space docking mechanism's working process can be roughly divided into five stages, including docking preparation, docking with buffer, capturing with electromagnet lock, pulling back of the docking ring and locking with the docking lock. If passive compliance control method is adopted at the second stage while the environmental situation is not completely known, the performance of the docking system can be unstable and high impact may occur because of the system's poor robustness. Therefore, using active compliance control method by measuring the force signal of the docking mechanism is of great practical significance.

Impedance controldoes not control the desire position or force directly. Instead, it achieves the purpose of compliance control by establishing and regulating the dynamic relationship between robot's contact force and position revision. By detecting the actual position of the actuator, we can get the contact force with the help of the environment rigidity. After comparing it with expected contact force, we can obtain the revised position by using the target impedance. Then, with the help of robot inverse kinematics and trajectory planning, the position of robot's end actuator is changed to reduce the contact force. This control method has great robustness under disturbed and uncertain environment, which can be quite useful.

In this paper, a highly effective multi-axis force measurement system is built to acquire and analyze the real time force from six separate force sensors. According to the data analysis of the measurement system, a compound contact force is used as the feedback signal to control the movement of the space docking mechanism. At last, after a brief introduction to the ground-built docking test system, data analysis of contact force is presented based on the practical experiments.

\section{Configuration of Force Sensor in the Docking Mechanism}

The active part of the 6-UPS space docking mechanism is a 6-DOF parallel manipulator. Six Hooke hinges on the mobile platform are linked correspondingly to six equivalent spherical hinges on the fixed platform with six lead screws, and there are six separate single axis force sensor in the whole driving chain, as shown in Fig. 1. In the meantime, there are guiding petals and electromagnets mounted uniformly on the mobile platform, and the passive docking ring is just isomorphic. 


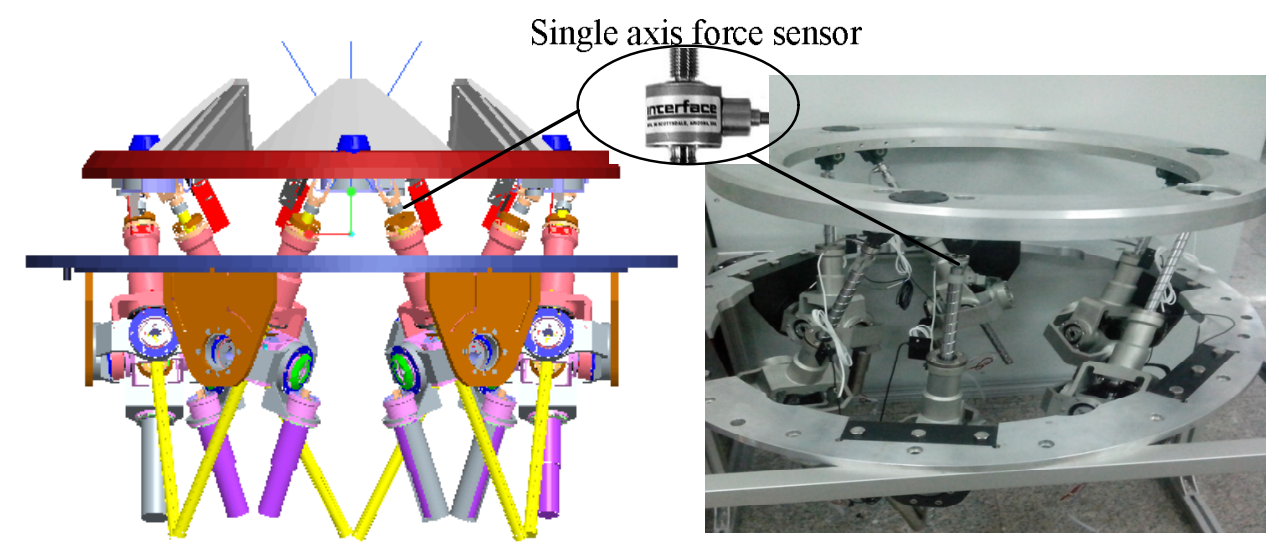

Fig.1: Configuration of Force Sensor in 6-UPS Space Docking Mechanism

Each prismatic pair of the 6-UPS docking mechanism is driven by a brush DC motor via a reducer and a set of lead screw, which provides a large reduction ratio. And the rotary motion of motor turns into linear motion after this transformation. Meanwhile, six force sensors are mounted between the Hooke hinges and screws in order to measure the axial force.

\section{Load Calculating from Six Force Sensors}

In order to get the six-dimensional contact force $\left(F_{x}, F_{y}, F_{z}, M_{x}, M_{y}, M_{z}\right)^{\mathrm{T}}$ to be used in motion controller, load calculating must be carried on to translate the six separate forces from six lead screw to an integral six-dimensional contact force.

The calculating process can be divided into two steps, in the coordinate system $O_{0} x_{0} y_{0} z_{0}$. First, coupling the tension or pressure from six separate axis to the point $O_{0}$; Secondly, transform the compound six-dimensional contact force from point $O_{0}$ to point $O$ 。

Suppose the single-dimensional contact force in the branched chain No i is $\mathscr{F}_{i}$, and it can be expressed as $\stackrel{\mathbf{W}}{F_{i}}=F_{i} \cdot \frac{\vec{l}_{i}}{\left|l_{i}\right|}$. Record $\stackrel{\mathbf{v}}{l_{i}}=\left(x_{i}, y_{i}, z_{i}, 0\right)^{\mathrm{T}}$, and decompose $\stackrel{\mathbf{w}}{F_{i}}$ in direction $x_{0}, y_{0}$ and $z_{0}$ under the coordinate system $O_{0} x_{0} y_{0} z_{0}$, as shown in Fig.2.

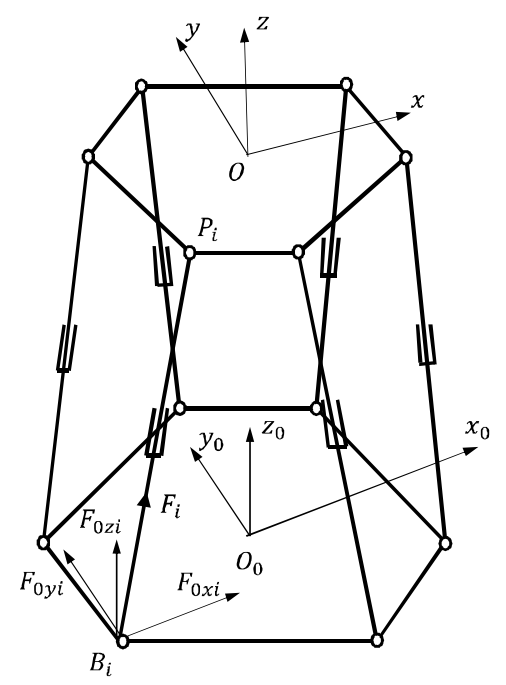

Fig.2: Force Calculating of the Space Docking Mechanism

$F_{i}$ in direction $x_{0}, y_{0}$ and $z_{0}$ can be expressed as: 


$$
\left\{\begin{array}{l}
F_{0 x i}=\frac{x_{i}}{\left|l_{i}\right|} \cdot F_{i}=\frac{x_{i}}{\sqrt{x_{i}^{2}+y_{i}^{2}+z_{i}^{2}}} \cdot F_{i}=\frac{x_{i}}{l_{0}+\Delta l_{i}} \cdot F_{i} \\
F_{0 y i}=\frac{y_{i}}{\left|l_{i}\right|} \cdot F_{i}=\frac{y_{i}}{\sqrt{x_{i}^{2}+y_{i}^{2}+z_{i}^{2}}} \cdot F_{i}=\frac{y_{i}}{l_{0}+\Delta l_{i}} \cdot F_{i} \\
F_{0 z i}=\frac{z_{i}}{\left|l_{i}\right|} \cdot F_{i}=\frac{z_{i}}{\sqrt{x_{i}^{2}+y_{i}^{2}+z_{i}^{2}}} \cdot F_{i}=\frac{z_{i}}{l_{0}+\Delta l_{i}} \cdot F_{i}
\end{array}\right.
$$

Translate the three component $\left(F_{0 x i}, F_{0 y i}, F_{0 z i}\right)$ to point $O_{0}$, and then we sum the force and torque in all directions, a six-dimentional force in point $O_{0}$ can be described as :

$$
\left\{\begin{array}{l}
F_{0 x}=\sum_{i=1}^{6} F_{0 x i}=\sum_{i=1}^{6} \frac{x_{i}}{\sqrt{x_{i}^{2}+y_{i}^{2}+z_{i}^{2}}} F_{i}=\sum_{i=1}^{6} \frac{x_{i}}{l_{0}+\Delta l_{i}} F_{i} \\
F_{0 y}=\sum_{i=1}^{6} F_{0 y i}=\sum_{i=1}^{6} \frac{y_{i}}{\sqrt{x_{i}^{2}+y_{i}^{2}+z_{i}^{2}}} F_{i}=\sum_{i=1}^{6} \frac{y_{i}}{l_{0}+\Delta l_{i}} F_{i} \\
F_{0 z}=\sum_{i=1}^{6} F_{0 z i}=\sum_{i=1}^{6} \frac{z_{i}}{\sqrt{x_{i}^{2}+y_{i}^{2}+z_{i}^{2}}} F_{i}=\sum_{i=1}^{6} \frac{z_{i}}{l_{0}+\Delta l_{i}} F_{i} \\
M_{0 x}=\sum_{i=1}^{6} F_{0 z i} r_{2} \sin \varphi_{i}=r_{2} \sum_{i=1}^{6} \frac{z_{i}}{\sqrt{x_{i}^{2}+y_{i}^{2}+z_{i}^{2}}} F_{i} \sin \varphi_{i}=r_{2} \sum_{i=1}^{6} \frac{z_{i}}{l_{0}+\Delta l_{i}} F_{i} \sin \varphi_{i} \\
M_{0 y}=-\sum_{i=1}^{6} F_{0 z i} r_{2} \cos \varphi_{i}=-r_{2} \sum_{i=1}^{6} \frac{z_{i}}{\sqrt{x_{i}^{2}+y_{i}^{2}+z_{i}^{2}}} F_{i} \cos \varphi_{i}=-r_{2} \sum_{i=1}^{6} \frac{z_{i}}{l_{0}+\Delta l_{i}} F_{i} \cos \varphi_{i} \\
M_{0 z}=\sum_{i=1}^{6}\left(F_{0 y i} r_{2} \cos \varphi_{i}-F_{0 x i} r_{2} \sin \varphi_{i}\right)=r_{2} \sum_{i=1}^{6} \frac{y_{i} \cos \varphi_{i}-x_{i} \sin \varphi_{i}}{\sqrt{x_{i}^{2}+y_{i}^{2}+z_{i}^{2}}} F_{i}=r_{2} \sum_{i=1}^{6} \frac{y_{i} \cos \varphi_{i}-x_{i} \sin \varphi_{i}}{l_{0}+\Delta l_{i}} F_{i}
\end{array}\right.
$$

In order to get the real force to be used in motion controller, the above deduced contact force should be transformed to point $O$ on mobile platform from point $O_{0}$ as follows :

$$
\left\{\begin{array}{l}
F_{x}=F_{o x} \\
F_{y}=F_{o y} \\
F_{z}=F_{o z} \\
M_{x}=F_{o y} p_{z}-F_{0 z} p_{y}+M_{o x} \\
M_{y}=F_{o z} p_{x}-F_{0 x} p_{z}+M_{o y} \\
M_{z}=F_{o x} p_{y}-F_{0 y} p_{x}+M_{o z}
\end{array}\right.
$$

Besides, the plane of point $O$ is the winch point, and it is different from the real contact surface with an offset $h$ in direction $z$, so corrections can be expressed as :

$$
\left\{\begin{array}{l}
p_{\text {newx }}=h(\sin \alpha \sin \gamma+\cos \alpha \sin \beta \cos \gamma)+p_{x} \\
p_{\text {newy }}=h(-\sin \alpha \cos \gamma+\cos \alpha \sin \beta \sin \gamma)+p_{y} \\
p_{\text {newz }}=h \cdot \cos \alpha \cos \beta+p_{x}
\end{array}\right.
$$

The final transformation after correction can be expressed as matrix : 


$$
\left[\begin{array}{c}
F_{x} \\
F_{y} \\
F_{z} \\
M_{x} \\
M_{y} \\
M_{z}
\end{array}\right]=\left[\begin{array}{cccccc}
1 & 0 & 0 & 0 & 0 & 0 \\
0 & 1 & 0 & 0 & 0 & 0 \\
0 & 0 & 1 & 0 & 0 & 0 \\
0 & p_{\text {newz }} & -p_{\text {newy }} & 1 & 0 & 0 \\
-p_{\text {newz }} & 0 & p_{\text {newx }} & 0 & 1 & 0 \\
p_{\text {newy }} & -p_{\text {newx }} & 0 & 0 & 0 & 1
\end{array}\right]\left[\begin{array}{c}
F_{0 x} \\
F_{0 y} \\
F_{0 z} \\
M_{0 x} \\
M_{0 y} \\
M_{0 z}
\end{array}\right]
$$

\section{Structure of Measurement System}

The control system of the docking mechanism is made up of one remote control computer, one master computer, one motion controller, one DAQ card, six force sensors, six drivers and six DC brush motors, as shown in Fig. 3.

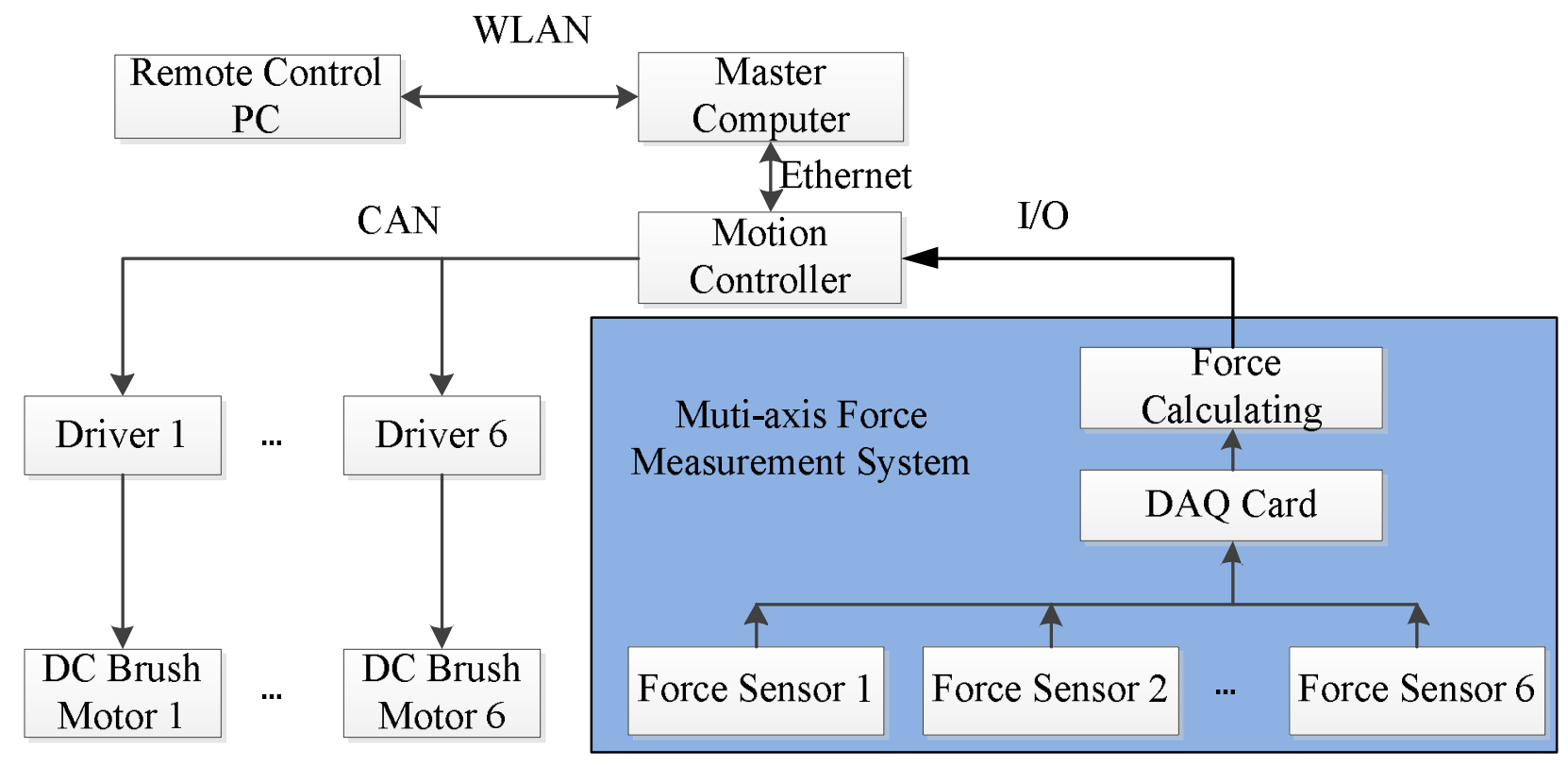

Fig.3: Structure of Measurement System in the whole Control System

The DAQ card sends force signal measured by six force sensors to the master computer via the motion controller. By using the force signal with impedance control method, the position revision of the active docking ring can be calculated. After this, motors' rotational turns can be calculated via inverse kinematics, and then be sent to six drivers via the motion controller, driving the DC brush motors to the right position via PID position control.

\section{Experiment Results}

Experimental Equipment. Space docking experiment is conducted with the ground-built docking test system which is made up of 6-UPS docking system and ground-built air floatation system, as shown in Fig. 4. The active part and passive part of the 6-UPS docking mechanism are mounted respectively in the corresponding parts of ground-built air floatation device. The ground-built air floatation system can provide buoyancy to offset the gravity of the whole device in order to simulate weightless environment in space.

Experimental Process and Result Analysis. After programming the Cartesian impedance control program with the parameter combination mentioned above, docking experiment is conducted on the ground-built docking test platform. The changing of time-varying contact force and position error in direction $\mathrm{Z}$ during the docking process is shown in Fig. 5.

According to the experimental figures, the contact force during the docking process can be controlled under $200 \mathrm{~N}$ with the help of impedance control method, and it can be stable at $0 \mathrm{~N}$ in the end. 
And the position change in $\mathrm{Z}$ direction is quite stable. In short, this method can greatly ensure the security buffer of the 6-UPS space docking mechanism, which is significant in practice.

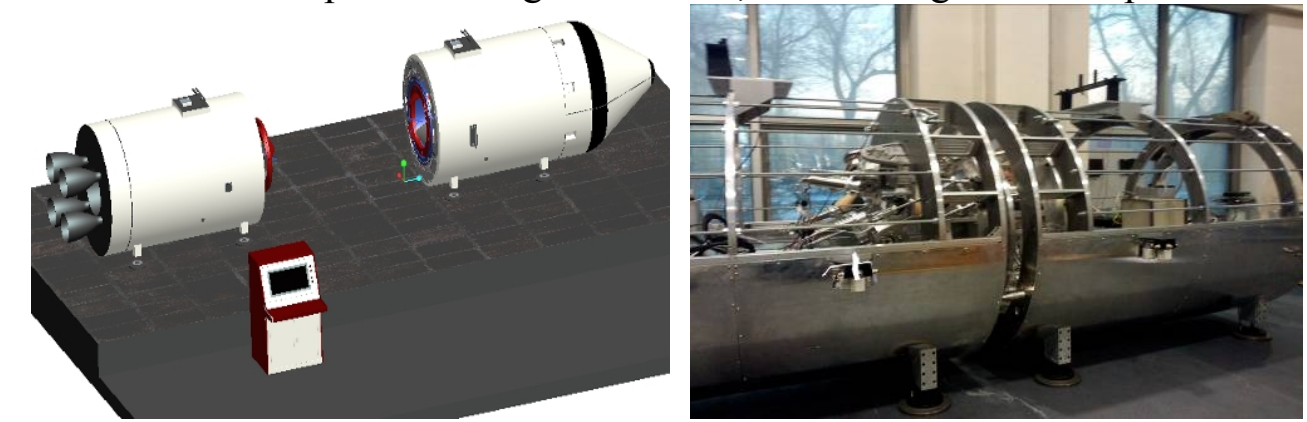

Fig.4: Ground-built Docking Test System
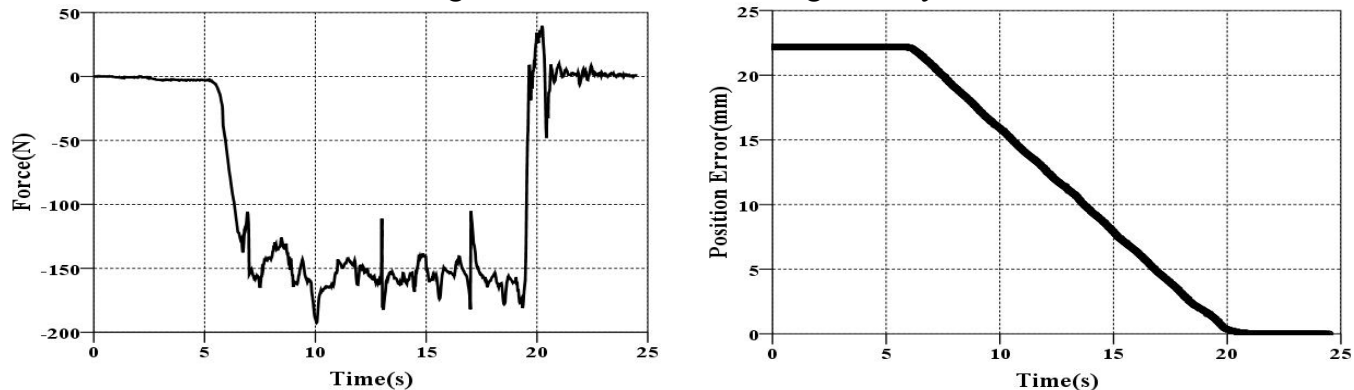

Fig.5: Contact Force and Position Error of Docking Experiment

\section{Conclusion}

In this paper, a highly effective multi-axis force measurement system based on the feedback signal from 6 separate force sensors is presented. Using the force parameters calculated in the measurement system, this motion controller can tremendously improve the buffer effect at the docking stage during the experiment. It is recommendable for future researchers to improve the robustness of the control system in case of the environmental mutation condition ${ }^{[5]}$, which can expand the application field of this method.

\section{Acknowledgement}

This work is supported by National High-tech R\&D Program of China (No.2014AA041601) and China Postdoctoral Science Foundation (No.2014M561338).

\section{References}

[1] Salisbury, J. Kenneth. "Active stiffness control of a manipulator in Cartesian coordinates." Decision and Control including the Symposium on Adaptive Processes, 1980 19th IEEE Conference on. Vol. 19. IEEE, 1980.

[2] Maples, J., and Joseph Becker. "Experiments in force control of robotic manipulators." Robotics and Automation. Proceedings. 1986 IEEE International Conference on. Vol. 3. IEEE, 1986.

[3] Blauer, Michael, and P. I. E. R. R. E. R. Belanger. "State and parameter estimation for robotic manipulators using force measurements." Automatic Control, IEEE Transactions on 32.12 (1987): 1055-1066.

[4] Kazanzides, Peter, N. Scott Bradley, and W. Wolovich. "Dual-drive force/velocity control: implementation and experimental results." Robotics and Automation, 1989. Proceedings., 1989 IEEE International Conference on. IEEE, 1989.

[5] Merlet, J. P. "C-surface applied to the design of an hybrid force-position robot controller." Robotics and Automation. Proceedings. 1987 IEEE International Conference on. Vol. 4. IEEE, 1987. 